\title{
A treatment of the exclusion volume term in the inhomogeneous Poisson-Boltzmann theory for an ion-dipole mixture
}

\author{
C.W.Outhwaite ${ }^{1}$, S.Lamperski $^{2}$ \\ 1 Department of Applied Mathematics, University of Sheffield, \\ Sheffield, S3 7RH, UK \\ 2 Department of Physical Chemistry, Faculty of Chemistry, \\ A.Mickiewicz University, \\ Grunwaldzka 6, 60-780 Poznan, Poland
}

Received August 8, 2001

\begin{abstract}
The exclusion volume approximation in the Poisson-Boltzmann theory is analysed at the mean field level for an ion-dipole mixture against a plane, uniformly charged hard wall. An earlier treatment is extended to take account of the deviation of the exclusion volume term from the uncharged wall - uncharged hard sphere distribution function. Preliminary numerical results are presented for a $1: 1$ electrolyte at $c=1.0 \mathrm{~mole} / \mathrm{dm}^{3}$ with unequal ion and dipole sizes.
\end{abstract}

Key words: excluded volume term, Poisson-Boltzmann, ion-dipole mixture, electric double layer

PACS: $68.08 .-p$

\section{Introduction}

The electric double layer, in the neighbourhood of a charged surface in an electrolyte solution, plays a key role in understanding the behaviour of many physical, chemical and biological phenomena. The classical treatment of the electrical double layer is based on the mean field Poisson-Boltzmann (PB) equation where the electrolyte solution is modelled by a system of point ions moving in a dielectric continuum. Many attempts have been made to improve both the electrolyte model and the theory of this classical approach. A basic reference electrolyte model is the primitive model (PM) where the ions in the dielectric are given finite size by being represented as charged hard spheres. More realistic models treat the solvent on an equal footing with the solute, the solvent having dipoles and higher order multipoles. 
Theoretical improvements have been based on improved or modified PB equations, integral equations and simulation $[1,2]$.

We consider here a PM electrolyte or an ion-dipole mixture against a uniformly charged plane hard wall. The emphasis will be upon examining the influence of ion or solvent size upon the structure of the electric double layer within a mean electrostatic potential framework. Various attempts have been made to account for the steric effect of the ions and the solvent. The earliest work was that of Stern [3] who introduced the idea of an inner compact layer to correct the Gouy-Chapman $[4,5]$ theory which overestimated ionic adsorption. Stern's approach has led to many different models and theories for the structure of the solution next to an electrode [6-8], but there are difficulties in reconciling many of these approaches with an overall consistent statistical mechanical picture of the double layer. An important related aspect is a realistic model of the electrode in assessing its influence on ionic distribution and adsorption $[9,10]$.

The first formal statistical mechanical analysis detailing the deficiences of the PB equation was due to Kirkwood [11]. He showed that the two approximations in the theory were the neglect of the exclusion volume term and of the fluctuation potential. The exclusion volume term arises from the discharged ion in the formal charging process, and in the double layer it plays a major role in controlling the surface concentration of the ions. The various treatments and relative importance of the two approximations within the mean electrostatic framework for the restricted PM (RPM) are given in $[1,12]$. There has been a recent resurgence of interest in incorporating steric effects into the $\mathrm{PB}$ equation in a simple fashion. These attempts are based on lattice model [13-15] and functional [16-18] approaches. The derivation in $[13,18]$ of these improved $\mathrm{PB}$ equations invokes solvent exclusion volume effects and the new theories impose an upper limit on the surface concentration of the counterions. No treatment is made of fluctuation effects and the solvent is solely considered as neutral hard spheres. The underlying electrolyte model, when the solvent is uncharged hard spheres moving in a dielectric continuum, is the solvent primitive model (SPM). The modified Poisson-Boltzmann (MPB) theory $[1,12,19$ 21 ] treats both the exclusion volume and the fluctuation potential term and formally prevents unphysical counterion surface coverage at high surface charge. By neglecting the fluctuation term, we show here how the MPB exclusion volume term reduces to expressions analogous to the recent improved PB results. Preliminary results are also presented for the ion-dipole mixture with different sizes which extends earlier work [22] on a corrected PB theory incorporating exclusion volume terms.

\section{Theory}

The electric double layer is modelled by a mixture of charged hard spheres and dipolar hard spheres moving in a medium of background relative electric permittivity $\varepsilon_{\infty}$ in the neighbourhood of a uniformly charged plane hard wall. At a normal distance $x_{1}$ from the electrode into the solution, Poisson's equation for the mean 
electrostatic potential $\psi=\psi(1)$ is

$$
\frac{\mathrm{d}^{2} \psi}{\mathrm{d} x_{1}^{2}}=-\frac{1}{\varepsilon_{0} \varepsilon_{\infty}} \sum_{k} e_{k} n_{k} g_{k}+\frac{1}{\varepsilon_{0} \varepsilon_{\infty}} \frac{\mathrm{d} P}{\mathrm{~d} x_{1}}
$$

where

$$
P=n_{d} \int \mu \cdot \hat{x}_{1} g_{d} \mathrm{~d} \omega .
$$

Here $P=P(1)$ is the polarization with $\mathrm{d} \omega$ the dipole rotational elements, $e_{k}$ the charge on an ion of species $k, \mu$ the dipole moment, $n_{t}$ the bulk number density of species $t$ and $g_{k}, g_{d}$ the singlet distribution functions for an ion $k$ or dipole $d$ at $x_{1}$ respectively. The labels $s$ and $t$ are for either ions or dipoles while $k$ and $d$ signify ions and dipoles respectively. For both the PM and SPM, $P$ vanishes and $\varepsilon_{\infty}$ is replaced by the bulk relative electric permittivity (bulk dielectric constant) $\varepsilon$. However, in the PM, exclusion volume effects only arise through ion size while the SPM also incorporates hard sphere solvent effects.

Neglecting the fluctuation term [23],

$$
\begin{aligned}
& g_{k}=\zeta_{k} \exp \left(-\beta e_{k} \psi\right), \\
& g_{d}=\left(\zeta_{d} / 4 \pi\right) \exp (-\beta \mu \cdot \nabla \psi),
\end{aligned}
$$

where $\zeta_{k}=g_{k}\left(e_{k}=0\right), \zeta_{d}=g_{d}(\mu=0)$ are the exclusion volume terms. For high values of the surface charge $\sigma$, the exclusion volume terms will impose a limit on the surface concentration of the ions and solvent. The mean field approximation is given by replacing $\zeta_{k}, \zeta_{d}$ by the appropriate unit step functions.

To treat the exclusion volume terms, consider the exact equation (2.11) of [24],

$$
\ln g_{s}=-\beta \phi_{s}+\int_{0}^{1} \int \sum_{t} n_{t} c_{s t} \frac{\partial g_{t}}{\partial \lambda} \mathrm{d} 2 \mathrm{~d} \lambda
$$

where $\phi_{s}$ is the interaction between the particle $s$ and the field due to the electrode, $c_{s t}=c_{s t}(1,2 \mid \lambda)$ is the nonuniform direct correlation function and $\lambda$ is the coupling parameter for the electrode field. Although developed for the PM, equation (5) also holds for the hard sphere ion-dipole mixture when $\mathrm{d} 2$ includes the rotational elements for $t$ running over the dipole species.

The exclusion volume term from equation (5) is

$$
\ln \zeta_{s}=-\beta \phi_{s}(s=0)+\int_{0}^{1} \int \sum_{t} n_{t} c_{s t}(s=0) \frac{\partial g_{t}}{\partial \lambda} \mathrm{d} 2 \mathrm{~d} \lambda,
$$

where $s=0$ means that the ion or dipole at $x_{1}$ is discharged.

A formal decomposition of $c_{s t}$ into a short and long range part means we expect the long range term to vanish and $c_{s t}(s=0)$ to be of short range, independent of $\lambda$. Also $\phi(s=0)$ is simply the hard sphere - hard wall interaction. So integrating with respect to $\lambda$,

$$
\ln \zeta_{s}=\int \sum_{t} n_{t} c_{s t}(s=0)\left[g_{t}-1\right] \mathrm{d} 2
$$


with $\zeta_{s}=0$ when $x_{1}$ is less than the distance of closest approach of $s$ to the electrode. Now putting the surface charge zero in equation (7), and then subtracting the result from equation (7), gives

$$
\ln \left(\zeta_{s} / \zeta_{s}^{0}\right)=\int \sum_{t} n_{t}\left\{\left[c_{s t}(s=0) g_{t}-c_{s t}^{0} g_{t}(\sigma=0)\right]+\left[c_{s t}^{0}-c_{s t}(s=0)\right]\right\} \mathrm{d} 2,
$$

where $\zeta_{s}^{0}=\zeta_{s}(\sigma=0)$ and $c_{s t}^{0}$ indicates that in $c_{s t}$ both the molecule $s$ at $x_{1}$ and surface charge are discharged. Setting $c_{s t}(s=0)=c_{s t}^{0}$, which is true for the bulk, gives

$$
\ln \left(\zeta_{s} / \zeta_{s}^{0}\right)=\int \sum_{t} n_{t} c_{s t}^{0}\left[g_{t}-g_{t}(\sigma=0)\right] \mathrm{d} 2 .
$$

Equations similar to equation (9) can be derived from the other standard statistical mechanical theories [1, p. 188-189], although now $c_{s t}^{0}$ takes its bulk value rather than the inhomogeneous value in equation (9). Given a value for $c_{s t}^{0}$, such as the bulk Percus-Yevick (PY) value for a mixture of uncharged hard spheres [25], the equations (1), (3), (4), (9) form a consistent set of equations for $\psi$. We shall not consider the full solution here but only analyse how the exclusion volume term given by equation (9) may alter the PB equation. A solution of the system for $\zeta_{s}$ given by the PY uncharged hard sphere singlet distribution function has been treated elsewhere [22].

Alternatively an equation analogous to equation (8) for the PM can be derived from the BBGY hierarchy [19]

$$
\begin{aligned}
\ln \left(\zeta_{s} / \zeta_{s}^{0}\right)= & \int_{x_{1}}^{\infty} \sum_{t} n_{t} \int_{S_{12}} \hat{x} \cdot \hat{r}_{12}\left[g_{t} g_{s t}\left(e_{s}=0\right)\right. \\
& \left.-g_{t}(\sigma=0) g_{s t}\left(e_{s}=\sigma=0\right)\right] \mathrm{d} S_{2} \mathrm{~d} x_{2} .
\end{aligned}
$$

We first show how equation (7) in conjunction with equations (3), (4) relates to the earlier work of Borukhov et al. $[14,15]$. Consider a single electrolyte with charges $e_{i}, e_{j}$, with all the ions and dipoles having the same diameter $R$ and $c_{s t}(s=0)$ given by the bulk uncharged direct correlation function. In this special case we write $\zeta$ and $c^{0}$ for $\zeta_{s}$ and $c_{s t}^{0}$ respectively as they are the same for all the species.

Equation (7) then simplifies to

$$
\begin{aligned}
\ln \zeta=\int & \sum_{k} n_{k} c^{0}\left[\zeta \exp \left(-\beta e_{k} \psi\right)-1\right] \mathrm{d} 2 \\
& +\int\left(n_{d} / 4 \pi\right) c^{0}[\zeta \exp (-\beta \mu \cdot \nabla \psi)-1] \mathrm{d} 2 .
\end{aligned}
$$

We now assume that the exclusion volume term only deviates slightly from its bulk value of unity, and write $\zeta=1+\delta$ where $|\delta|<1$. So expanding to the first order in $\delta$ and approximating $\delta$ in the integrand by its mean value $\bar{\delta}$ at $x_{1}$, gives

$$
\delta=\bar{\delta} X+X-X^{b}
$$


where

$$
\begin{aligned}
X & =n_{i} F_{i}+n_{j} F_{j}+n_{d} G_{d}, \\
X^{b} & =\sum_{s} n_{s} \int c^{0} \mathrm{~d} 2, \\
F_{k} & =\int c^{0} \exp \left(-\beta e_{k} \psi\right) \mathrm{d} 2, \\
G_{d} & =\int c^{0} D \mathrm{~d} 2, \\
D & =(1 / 4 \pi) \int \exp (-\beta \mu \cdot \nabla \psi) \mathrm{d} \omega=\sinh \Theta / \Theta, \\
\Theta & =\mu \beta \mathrm{d} \psi / \mathrm{d} x_{2} .
\end{aligned}
$$

Equating $\bar{\delta}$ to $\delta$ and solving equation (12) gives

$$
\zeta=\frac{1-X^{b}}{1-X}
$$

which is implicit in the RPM work of Bell and Levine [26]. To see if (19) may predict a finite counterion $g_{i}$ for a large surface charge, we use the approximation $c^{0}=$ $-1+O(n), r<R$, and zero elsewhere, and so for $x_{1}>R / 2, F_{k} \sim-v \exp \left(-\beta e_{k} \psi\right)$, $G_{d} \sim-v \exp (-\beta \mu \cdot \nabla \psi)$, where $v=4 \pi R^{3} / 3$ or the truncated sphere for $R / 2<x_{1}<$ $R$. With these approximations

$$
\zeta=\frac{1+v \sum_{s} n_{s}}{1+v\left[\sum_{k} n_{k} \exp \left(-\beta e_{k} \psi\right)+n_{d} \exp (-\beta \mu \cdot \nabla \psi)\right]},
$$

thus for $\left|\beta e_{i} \psi\right| \gg 1$ with $-\beta\left(\mu \cdot \nabla \psi-e_{i} \psi\right) \ll 0$

$$
g_{i} \rightarrow \frac{1}{v n_{i}}\left(1+v \sum_{s} n_{s}\right)
$$

which is finite. Multiplying numerator and denominator of equation (20) by $1+X^{b}$ and neglecting terms of order $n^{2}$, then putting $n_{d}=0$ or $\mu=0$ gives the special cases of [14]. The corrected PB equation is given by equations (1), (3), (4), (19) and when $n_{d}=0$ we are working with the PM so that the correction arises solely from the ion sizes, and when $\mu=0$ the correction is within the SPM and comes from the solvent size. As stressed by Kralji-Iglic and Iglic [13], Borukhov et al. [14], an exclusion volume term imposes a limit on the surface ion concentration. The present formulation of the MPB theory $[1,20]$ for the RPM utilizes approximations to equation (10) and would be expected to prevent unphysical counterion adsorption. As yet there are no MPB numerical results for high surface charge.

A failure of result (19) or (20) is that when the ions and dipole are discharged the exclusion volume term is unity, as in the PB theory, rather than the uncharged hard sphere-hard wall distribution function. Indeed this uncharged distribution function 
must contain important steric effects at high electrolyte concentrations. To overcome this deficiency we generalize the derivation of (19) by writing $\zeta_{s}=\zeta_{s}^{0}\left(1+\delta_{s}\right)$ where we assume $\left|\delta_{s}\right|$ is small. So, substituting in equation (9) for $\zeta_{s}$, using (3), (4) for the singlet distribution functions, expanding to first order in $\delta_{s}$, and approximating $\delta_{s}$ under the integral sign by its mean value $\bar{\delta}_{s}$ at $x_{1}$ gives

$$
\delta_{s}=\sum_{k} \bar{\delta}_{k} n_{k} F_{s k}+\bar{\delta}_{d} n_{d} G_{s d}+X_{s}-X_{s}^{0}
$$

where

$$
\begin{aligned}
X_{s} & =\sum_{k} n_{k} F_{s k}+n_{d} G_{s d} \\
X_{s}^{0} & =X_{s}(\sigma=0), \\
F_{s k} & =\int c_{s k}^{0} \zeta_{k}^{0} \exp \left(-\beta e_{k} \psi\right) \mathrm{d} 2, \\
G_{s d} & =\int c_{s d}^{0} \zeta_{d}^{0} D \mathrm{~d} 2 .
\end{aligned}
$$

If we approximate $\bar{\delta}_{s}$ by $\delta_{s}$ then equation (22) becomes a system of linear equations which can readily be solved for $\delta_{s}$. When $\sigma \rightarrow 0, \delta_{s} \rightarrow 0$ and so as required $\zeta_{s} \rightarrow \zeta_{s}^{0}$. For a single electrolyte with equal size ions of charge $e_{i}$ and $e_{j}$, simplifications occur as then for $c_{i i}^{0}=c_{i j}^{0}=c_{j j}^{0}, F_{i i}=F_{j i}, F_{j j}=F_{i j}$ and $G_{i d}=G_{j d}$. Furthermore if the dipoles are also the same size as the ions, $F_{d i}=F_{i i}, F_{d j}=F_{j j}, G_{i d}=G_{j d}=G_{d d}$. In particular for a $z: z$ electrolyte for equisized ions and dipoles,

$$
\zeta=\zeta^{0} \frac{1-n\left(F_{i i}^{0}+F_{j j}^{0}\right)-n_{d} G_{d d}^{0}}{1-n\left(F_{i i}+F_{j j}\right)-n_{d} G_{d d}},
$$

where $n_{i}=n_{j}=n$; and for the $1: z$ case with equal sizes,

$$
\zeta=\zeta^{0} \frac{1-n\left(z F_{i i}^{0}+F_{j j}^{0}\right)-n_{d} G_{d d}^{0}}{1-n\left(z F_{i i}+F_{j j}\right)-n_{d} G_{d d}},
$$

where $n_{i}=z n_{j}=z n$ and $\zeta=\zeta_{s}, \zeta^{0}=\zeta_{s}^{0}$. As before these two results are analogous to those of Borukhov et al.. In any investigation of the influence of the exclusion volume term given by equation (9), we would expect equation (22) to provide a reasonable first approximation to $\zeta_{s}$ for the numerical solution of the corrected PB equation, or the corresponding MPB theory in the RPM. In (22) the $\zeta_{s}^{0}$ and $c_{s t}^{0}$ can be estimated by any appropriate uncharged hard wall - hard sphere distribution function and bulk hard sphere direct correlation function respectively.

\section{Results and discussion}

We now present some preliminary results for the unequal size ion-dipole mixture based on equation (9) for $\zeta_{k}$ and equation (22) for $\zeta_{d}$. From equation (22), with 
$\delta_{k}=0$, we obtain

$$
\zeta_{d}=\zeta_{d}^{0} \frac{1+\sum_{k} n_{k}\left(F_{d k}-F_{d k}^{0}\right)-n_{d} G_{d d}^{0}}{1-n_{d} G_{d d}} .
$$

For consistency the dipole exclusion volume term should also be calculated from equation (9). The present numerical procedure needs to be adapted to solve this consistent situation, but we expect equation (29) to be a good approximation to $\zeta_{d}$ for the considered parameter range. The exclusion volume term for zero surface charge $\zeta_{s}^{0}$, and the uncharged bulk direct correlation function, are approximated by the corresponding Percus-Yevick values for uncharged hard sphere mixtures [25]. The three component PY $g_{s}^{0}$ has been given elsewhere [22] and the PY $c_{s t}$ can be derived from Baxter's work [27], namely

$$
r c_{i j}= \begin{cases}-\alpha_{i} r-\beta_{i}+2 \pi \sum_{s} n_{s} I_{s}, & r<R_{i j} \\ 0, & r>R_{i j}\end{cases}
$$

where

$$
\begin{gathered}
I_{s}=I_{b}=-\frac{R_{i}^{2}}{24}\left[12 \beta_{s}^{2}+12 \alpha_{s} \beta_{s}\left(r+R_{s}\right)+\alpha_{s}^{2}\left(2 R_{i} r+6 R_{s} r+3 R_{s}^{2}\right)\right], \quad r<S_{j i}, \\
I_{s}=I_{b}+\left(S_{i j}+r\right)^{2}\left\{\frac{\beta_{s}^{2}}{2}+\frac{\alpha_{s} \beta_{s}}{2}\left(R_{i s}+R_{j s}\right)+\frac{\alpha_{s}^{2}}{24}\left[r\left(2 S_{i j}-r\right)+3\left(R_{i j}+R_{s}\right)^{2}\right]\right\} \\
R_{i j}>r>S_{j i},
\end{gathered}
$$

and

$$
\alpha_{i}=\frac{1-\xi_{3}+3 R_{i} \xi_{2}}{\left(1-\xi_{3}\right)^{2}}, \quad \beta_{i}=-\frac{3 R_{i}^{2} \xi_{2}}{2\left(1-\xi_{3}\right)^{2}}, \quad \xi_{j}=\frac{\pi}{6} \sum_{i} n_{i} R_{i}^{3} R_{i}^{j} .
$$

Here $R_{i}$ is the diameter of $i$ with $R_{i j}=\left(R_{i}+R_{j}\right) / 2$, and $S_{i j}=\left(R_{i}-R_{j}\right) / 2$.

The numerical solution of Poisson's equation (1), in conjunction with equations $(3),(4)$ and exclusion volumes given by equation (9) for the ions and (29) for the dipoles, was obtained by a modification of the quasi-linearization technique of [22]. Calculations were performed for a 1:1 electrolyte at concentration $c=1.0 \mathrm{~mole} / \mathrm{dm}^{3}$ with $R_{+}=3.6 \AA, R_{-}=3.0 \AA$ while the solvent parameters were $R_{d}=2.4 \AA, \mu=$ $1.84 \mathrm{D}$ and concentration $c_{d}=45 \mathrm{~mole} / \mathrm{dm}^{3}$. The background permittivity $\varepsilon_{\infty}$, which reflects the atomic and electronic polarization of solute and solvent, was taken to be 2 and temperature $T=298.15 \mathrm{~K}$.

In the figures, comparisons are made with the case when the exclusion volume term is simply given by the PY uncharged singlet distribution function $g_{s}^{0}$, this situation being denoted by $g_{s}^{*}$. Figure 1 indicates a large reduction in the value of $\zeta_{k}$ from the PY $g_{k}^{0}$ at $\sigma=0.1 \mathrm{C} / \mathrm{m}^{2}$ in the vicinity of the electrode. In contrast there is only a small reduction in $\zeta_{d}$, so now it is the largest, not the smallest, exclusion volume term near the electrode. The small reduction in $\zeta_{d}$ is significant as it indicates a correlation with the surface charge which will become more important as $\sigma$ increases. A similar overall picture occurs for $\sigma=-0.1 \mathrm{C} / \mathrm{m}^{2}$.

The variation of the contact values of $\zeta_{s}$ and $g_{s}$ for $\sigma$ varying from -0.15 to $0.15 \mathrm{C} / \mathrm{m}^{2}$ are given in figures $2-4$. For the ions $\zeta_{k}$ is less than the PY $g_{k}^{0}$, except 


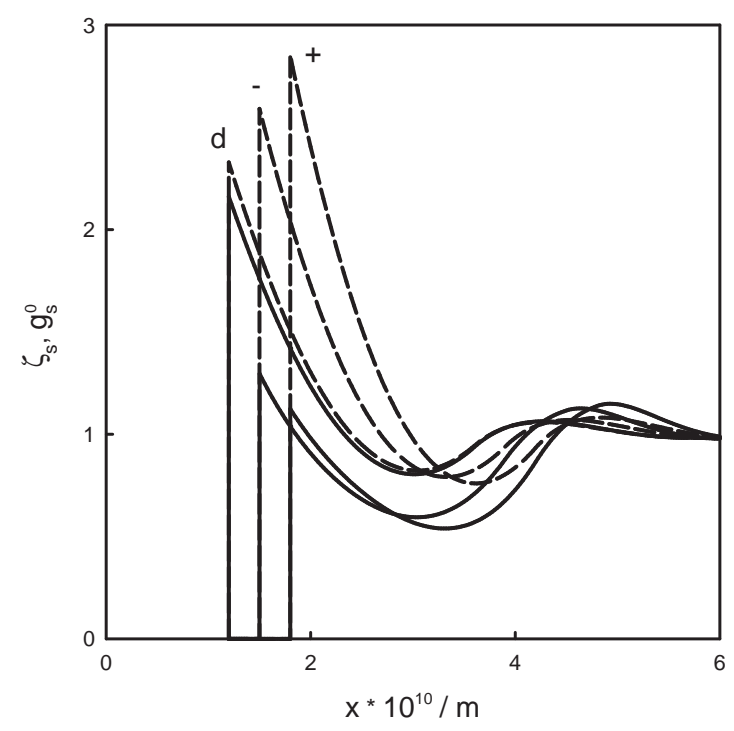

Figure 1. Exclusion volume term $\zeta_{s}$ (solid line) and PY $g_{s}^{0}$ (dashed line) at $\sigma=0.1 \mathrm{C} / \mathrm{m}^{2}$. Other parameters as in text. The solvent, anion and cation functions are denoted by $d,-,+$ respectively.

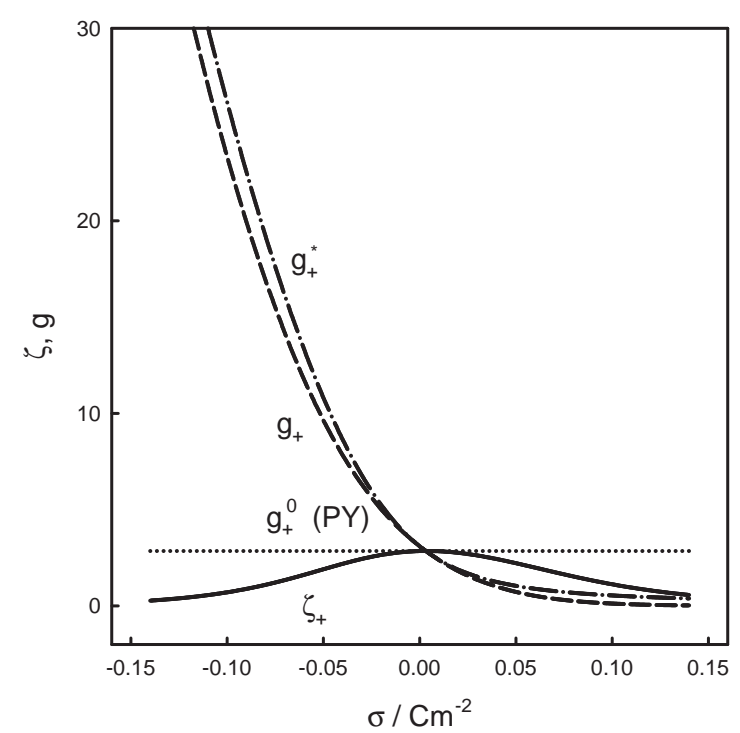

Figure 3. Contact value of the cation exclusion volume term and singlet function for $|\sigma|<0.15 \mathrm{C} / \mathrm{m}^{2}$. The singlet functions $g_{+}, g_{+}^{*}$ are calculated using $\zeta_{+}$and PY $g_{+}^{0}$ respectively.

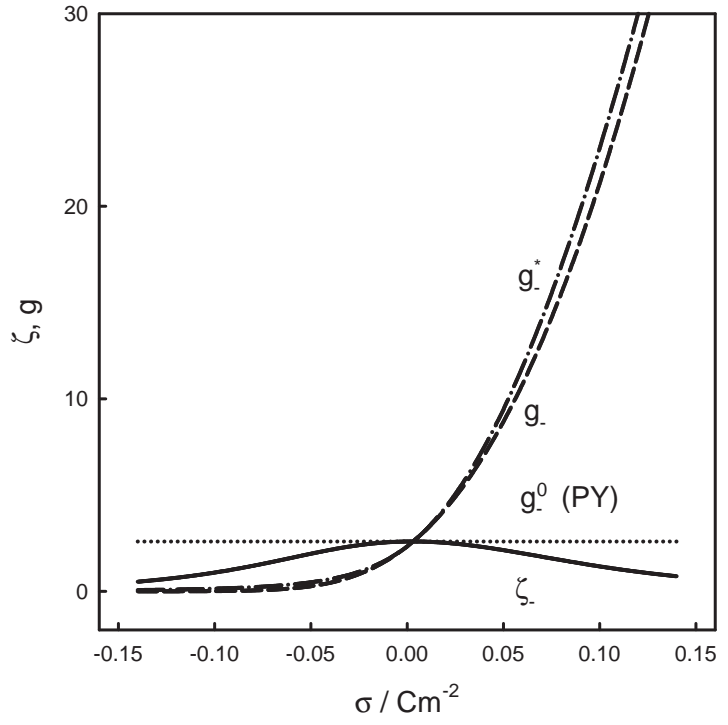

Figure 2. Contact value of the anion exclusion volume term and singlet function for $|\sigma|<0.15 \mathrm{C} / \mathrm{m}^{2}$. The singlet functions $g_{-}, g_{-}^{*}$ are calculated using $\zeta_{-}$and PY $g_{-}^{0}$ respectively.

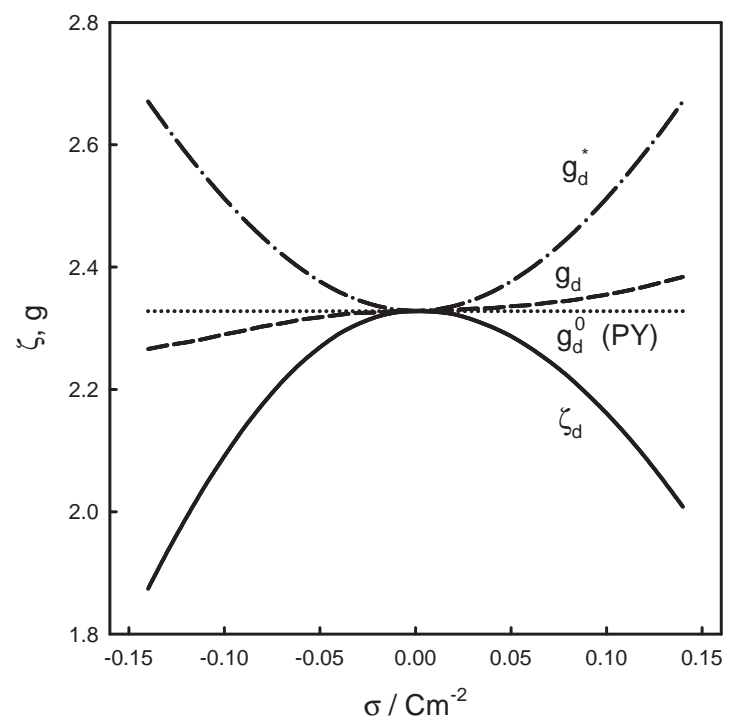

Figure 4. Contact value of the solvent exclusion volume term and singlet function for $|\sigma|<0.15 \mathrm{C} / \mathrm{m}^{2}$. The singlet functions $g_{d}, g_{d}^{*}$ are calculated using $\zeta_{d}$ and PY $g_{d}^{0}$ respectively. 


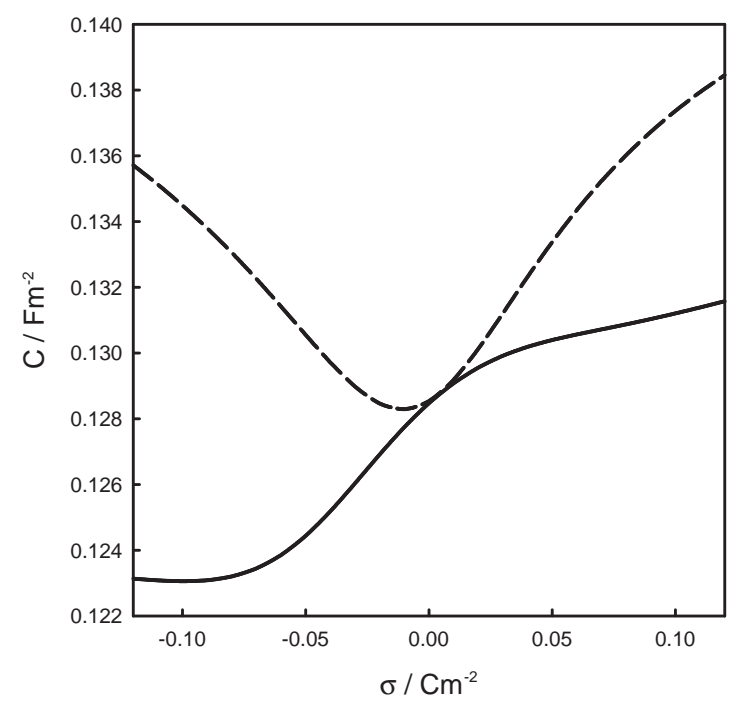

Figure 5. The differential capacitance $C$. The dashed line is the capacitance when the exclusion volume term is given by the PY $g_{s}^{0}$. when $\sigma=0$. This in turn reduces the contact value of $g_{k}$ for $\sigma \neq 0$ from $g_{k}^{*}$. However for the solvent when $\sigma>0$, the contact value of $g_{d}$ is larger than that of $g_{d}^{*}$ in contrast to its lower value when $\sigma<0$. This is a reflection of the different molecular sizes, with the dipole being the smallest. At these low surface charges the larger counterion (cation for $\sigma<0$ ) reduce the adsorption of the solvent molecules relative to the $g_{d}^{0}$, and the smaller counterion (anion for $\sigma>0$ ) increase the solvent adsorption. The effect is clearly seen in figure 5 for the differential capacitance. Rather than an asymmetric ' $\mathrm{U}$ ' shape from the $g_{s}^{*}$ as found in [22], the capacitance begins to adopt a shape reminiscent of group 2 of Parson's classification [28].

The absolute values of the capacitance are too low, and this can be attributed to many factors, two of the most probable being the poor representation of the solvent and the neglect of the fluctuation term in the distribution functions. However it appears that the formulation of the exclusion volume terms presented here which takes into account surface charge correlations, married with treating solute and solvent molecules on an equal footing, may prove fruitful. Work is in progress studying the implication of various parameter variations.

\section{References}

1. Carnie S.L., Torrie G.M. // Advances in Chemical Physics, 1984, vol. 56, p. 141.

2. Blum L., Henderson D. Fundamentals of Inhomogeneous Fluids (ed. D. Henderson). Marcel Dekker, 1992, p. 239.

3. Stern O.Z. // Electrochem., 1924, vol. 30, p. 508.

4. Gouy M.G.J. // Phys. France, 1910, vol. 9, p. 457.

5. Chapman D.L. // Philos. Mag., 1913, vol. 25, p. 475.

6. Fawcett W.R. // Israel J. Chemistry, 1979, vol. 18, p. 3.

7. Parsons R. // Chem. Rev., 1990, vol. 90, p. 813.

8. Lyklema J. Fundamentals of Interface and Colloid Science, vol. II. Academic Press, 1995.

9. Badiali J.P. // Electrochimica Acta, 1986, vol. 31, p. 149.

10. Guidelli R., Schmickler W. // Electrochimica Acta, 2000, vol. 45, p. 2317.

11. Kirkwood J.G. // J. Chem. Phys., 1934, vol. 2, p. 767.

12. Outhwaite C.W. Statistical Mechanics, vol. II. Specialist Periodical Report. London, Chemical Society, 1975, p. 188.

13. Kralj-Iglic V., Iglic A. // J. Phys. II France, 1996, vol. 6, p. 477. 
14. Borukhov I., Andelman D., Orland H. // Phys. Rev. Lett., 1997, vol. 79, p. 435.

15. Borukhov I., Andelman D., Orland H. // Electrochimica Acta, 2000, vol. 46, p. 221.

16. Lue L., Zoeller N., Blankschtein D. // Langmuir, 1999, vol. 15, p. 3726.

17. Trizac E., Raimbault J-L. // Phys. Rev. E, 1999, vol. 60, p. 6530.

18. Hansen J.P., Goulding D., van Roij R. // J. Phys. IV France, 2000, vol. 10, p. 27.

19. Outhwaite C.W., Bhuiyan L.B. // J. Chem. Soc., Far. Trans. 2, 1982, vol. 78, p. 775.

20. Outhwaite C.W., Bhuiyan L.B. // J. Chem. Soc., Far. Trans. 2, 1983, vol. 79, p. 707.

21. Lamperski S., Outhwaite C.W., Bhuiyan L.B. // Mol. Phys., 1996, vol. 87, p. 1049.

22. Lamperski S., Outhwaite C.W. // J. Electroanal. Chem., 1999, vol. 460, p. 135.

23. Outhwaite C.W. // Mol. Phys., 1983, vol. 48, p. 599.

24. Carnie S.L., Chan D.Y.C., Mitchell D.J., Ninham B.W. // J. Chem. Phys., 1981, vol. 74 , p. 1472.

25. Lebowitz J.L. // Phys. Rev. A, 1964, vol. 133, p. 895.

26. Levine S., Bell G.M. // J. Phys. Chem., 1960, vol. 64, p. 1188.

27. Baxter R.J. // J. Chem. Phys., 1970, vol. 52, p. 4559.

28. Parsons R. // Electrochimica Acta, 1976, vol. 21, p. 681.

\title{
Трактування члена виключеного об'єму в неоднорідній теорії Пуассона-Больцмана для іонно-дипольної суміші
}

\author{
К.В.Аутвайт ${ }^{1}$, С.Ламперскі ${ }^{2}$ \\ 1 Відділення прикладної математики, Шефілдський університет, \\ Шефілд, Великобританія \\ 2 Інститут фізичної хімії, хімічний факультет, Університет А.Міцкевича, \\ Познань, Польща
}

\section{Отримано 8 серпня 2001 р.}

Наближення виключеного об'єму в теорії Пуассона-Больцмана аналізується на рівні середнього поля для іонно-дипольної суміші біля плоскої, однорідно зарядженої твердої стінки. Попередній розгляд $€$ розширений до врахування відхилення члена виключеного об'єму від функції розподілу незаряджена стінка - незаряджена тверда сфера. Попередні числові результати є представлені для електроліту $1: 1$ при $\mathrm{c}=1.0 \mathrm{моль} /$ дм $^{3}$ з нерівними розмірами іонів і диполів.

Ключові слова: член виключеного об'єму, Пуассон-Больцман, іонно-дипольна суміш, електричний подвійний шар

PACS: $68.08 .-p$ 